MAGNETOHYDRODYNAMICS Vol. 54 (2018), No. 1-2, pp. 167-171

DOI: $10.22364 / \mathrm{mhd} .54 .1-2.30$

\title{
STABILITY OF CAPILLARY-GRAVITY WAVES IN THE PRESENCE OF MAGNETIC SURFACTANT IN AN EXTERNAL MAGNETIC FIELD
}

\author{
A.V.Zhukov \\ Institute of Mechanics, Moscow State University, 117192 Moscow, Russia
}

The stability of the capillary-gravity wave motion on the horizontal free surface of a viscous incompressible fluid in the presence of a magnetic surfactant in an external magnetic field was studied. It is shown that for a normal as well as for a tangential external magnetic field the horizontal free liquid surface is unstable for a field strength exceeding some critical value that does not depend on the elastic constant of the surfactant film. However, for an oblique external magnetic field the stability of the free surface depends not only on the field value but also on the surfactant elastic constant.

1. Governing equations. Recently, new surfactant molecules with magnetic properties have been synthesized [1]. This makes possible the magnetic control of the fluid surface properties by sufficiently strong $(0.4 \ldots 1 \mathrm{~T})$ external magnetic fields. The surface tension tensor for these media is anisotropic and depends on the magnetic field strength. A similar dependence was found for magnetic fluid-water interfaces subjected to magnetic fields of moderate intensity $(60 \ldots 110 \mathrm{G})[2]$. We study the stability of the capillary-gravity wave motion on the horizontal free surface of a viscous incompressible fluid in the presence of a magnetic surfactant in an external magnetic field.

Let $D^{-}$be a domain occupied by a fluid, $D^{+}$is an external domain and $\Sigma$ is the interfacial boundary defined by the equation $z \equiv x^{3}=\eta\left(x^{a}\right), a=1,2$, where $x^{i}$ denotes the Cartesian coordinates:

$$
D^{-}(t): z<\eta\left(x^{a}, t\right), \quad D^{+}(t): z>\eta\left(x^{a}, t\right), \quad \Sigma(t): z=\eta\left(x^{a}, t\right) .
$$

In the equilibrium state $\eta=0$. The $z$-axis is directed upwards. The normal vector $\mathbf{n}$ at the boundary is defined as external normal to $D^{-}$.

Inside the domain $D^{-}$the Navier-Stokes equations are valid:

$$
\partial_{k} v^{k}=0, \quad \rho\left(\partial_{\mathrm{t}} v^{i}+v^{k} \partial_{k} v^{i}\right)=\partial_{k} p^{i k(f)}+\rho g^{i},
$$

where

$$
\mathbf{g}=(0,0,-g) ; \quad p_{i k}^{(f)} \equiv-p \delta_{i k}+\mu\left(\nabla_{i} v_{k}+\nabla_{k} v_{i}\right) .
$$

Here $\rho$ is the density and $\mu$ is the dynamic viscosity of the fluid, $v^{i}$ denotes the velocity components, $p$ is the pressure, $p_{i k}^{(f)}$ is the stress tensor (excluding the magnetic field stress) and $\mathbf{g}$ is the acceleration of gravity. Inside $D^{+} p=p_{0}=$ const.

The following conditions are valid at the interface [2]:

$$
\begin{gathered}
\partial_{\mathrm{t}} \eta-v^{3}+v^{a} \partial_{a} \eta=0 \\
{\left[H_{t a}\right]=-4 \pi \lambda_{\mathrm{n}} \nabla_{a}\left\langle B_{\mathrm{n}}\right\rangle, \quad\left[B_{\mathrm{n}}\right]=-4 \pi \lambda_{\mathrm{t}} \nabla_{a}\left\langle H_{\mathrm{t}}^{a}\right\rangle} \\
{\left[p_{b}^{i}\right] n_{i}-\nabla_{a} \sigma_{b}^{a}=0, \quad\left[p^{i k} n_{i} n_{k}\right]-\sigma^{a b} b_{a b}=0}
\end{gathered}
$$

where

$$
p_{i k}=p_{i k}^{(f)}-\frac{H^{2}}{8 \pi} \delta_{i k}+\frac{H_{i} H_{k}}{4 \pi}
$$




\section{A.V. Zhukov}

Here $\lambda_{\mathrm{n}}=\Gamma c_{\mathrm{n}}, \lambda_{\mathrm{t}}=\Gamma c_{\mathrm{t}}$ are the coefficients determining the surface magnetization of the surfactant film in normal and tangential directions, respectively: $\mathbf{m}_{\mathrm{n}}=-\lambda_{\mathrm{n}}\left\langle\mathbf{B}_{\mathrm{n}}\right\rangle, \mathbf{m}_{\mathrm{t}}=-\lambda_{\mathrm{t}}\left\langle\mathbf{H}_{\mathrm{t}}\right\rangle[2], \Gamma$ is the surface surfactant density, $\langle f\rangle \equiv\left(f_{+}+f_{-}\right) / 2,[f] \equiv f_{+}-f_{-} ; \mathbf{H}$ and $\mathbf{B}$ are the vectors representing the magnetizing field and the magnetic field, respectively, $p_{i k}$ stands for the components of the total stress tensor, $b_{a b}$ denotes the components of the second quadratic form of the boundary, and $\sigma_{a b}$ denotes the components of the surface tension tensor:

$$
\sigma_{a b}=\left(\gamma_{0}-\beta \Gamma-\frac{1}{2} \lambda_{\mathrm{n}}\left\langle B_{\mathrm{n}}\right\rangle^{2}+\frac{1}{2} \lambda_{\mathrm{t}} h_{c} h^{c}\right) a_{a b}-\lambda_{\mathrm{t}} h_{a} h_{b}, h_{a} \equiv\left\langle H_{t a}\right\rangle .
$$

The constant $\beta$ characterizes the dependence of the surface tension on the surfactant concentration [4]. The dependence of $\sigma_{a b}$ on the magnetic field strength is taken from [2]. The following stability conditions are necessary: $\lambda_{\mathrm{n}} \geq 0$ and $\lambda_{\mathrm{t}} \leq 0[3]$.

The dynamics of the surfactant surface density is described by the following equations:

$$
\partial_{\mathrm{t}} \Gamma+\nabla_{a}\left(\Gamma u_{\Sigma}^{a}\right)+\frac{1}{2} \Gamma a^{a b} \partial_{\mathrm{t}} a_{a b}=0 ; \quad u_{\Sigma}^{a}=v_{\mathrm{t}}^{a} .
$$

Here the surfactant diffusion effects and the mass transfer effects to or from the dividing surface are neglected and the surfactant surface velocity $u_{\Sigma}^{a}$ is assumed

equal to the tangential fluid velocity [4]; $a_{a b}$ stands the components of the first quadratic form on the surface.

The Maxwell's equations for the magnetic field in non-magnetizable media are

$$
\operatorname{div} \mathbf{H}=0, \quad \operatorname{rot} \mathbf{H}=0, \quad \mathbf{B}=\mathbf{H} .
$$

The conditions at infinity take the form

$$
z \rightarrow-\infty: \mathbf{v} \rightarrow 0 ; \quad z \rightarrow \pm \infty: \mathbf{H} \rightarrow\left(H_{\mathrm{t}}, 0, H_{\mathrm{n}}\right),
$$

where $H_{\mathrm{t}}$ and $H_{\mathrm{n}}$ are the given values of the external magnetic field strength in tangential and normal directions to the horizontal unperturbed surface.

2. Linearization and dispersion equations. To study capillary-gravity waves, we performed the linearization of Eqs. (1)-(11) with respect to the equilibrium solution

$$
\begin{gathered}
z>0: p=p_{0}, \mathbf{H}=\left(H_{\mathrm{t}}, 0, H_{\mathrm{n}}\right) ; \\
z<0: p=p_{0}-\rho g z, v^{i}=0, \mathbf{H}=\left(H_{\mathrm{t}}, 0, H_{\mathrm{n}}\right) ; \quad \eta=0 .
\end{gathered}
$$

Each of the perturbations may be represented as a superposition of normal modes, for example,

$$
p^{\prime}=p-p_{(\text {eq })}=\int \exp \left(s t+i k_{\alpha} x^{\alpha}\right) \hat{p}\left(k_{\alpha}, s, z\right) \mathrm{d}^{2} k, \quad \alpha=1,2 .
$$

Here $s=s\left(k_{1}, k_{2}\right)$ is the solution of the dispersion equation and the '(eq)' subscript denotes values taken from the equilibrium solution.

The linearization of Eqs. (1)-(11) give the following expressions for the Fourier coefficients of the perturbations:

$$
\begin{array}{cc}
\hat{v}_{3}=A \exp (k z)+C \exp (l z) ; & l^{2}=k^{2}+s / \nu, \\
\nu=\mu / \rho, \quad \operatorname{Re}(l)>0 ; & \hat{\eta}=(A+C) / s,
\end{array}
$$


Stability of capillary-gravity waves in the presence of magnetic surfactant in an ...

$$
\begin{gathered}
\hat{v}_{\alpha}=\frac{i k_{\alpha}}{k^{2}}(k A \exp (k z)+l C \exp (l z)), \\
\hat{p}=-\frac{\rho s A}{k} \exp (k z) ; \quad \hat{\Gamma}=\frac{\Gamma}{s}(k A+l C) ; \\
\hat{H}_{\alpha}=i k_{\alpha} \hat{h}^{ \pm} \exp (\mp k z), \quad \hat{H}_{3}=\mp k \hat{h}^{ \pm} \exp (\mp k z) ; \quad k=|\mathbf{k}| ; \\
\hat{h}^{+}+\hat{h}^{-}=\frac{4 \pi c_{\mathrm{t}} \Gamma H_{3} k \hat{\eta}}{1-2 \pi c_{\mathrm{t}} \Gamma k}, \quad \hat{h}^{+}-\hat{h}^{-}=-\frac{4 \pi i c_{\mathrm{n}} \Gamma H_{\alpha} k^{\alpha} \hat{\eta}}{1+2 \pi c_{\mathrm{n}} \Gamma k} .
\end{gathered}
$$

Using these expressions yields the dispersion equation

$$
\begin{aligned}
& -\left(\omega_{0}^{2}+2 \nu k l s\right)\left(2 \nu k^{2} s+\varepsilon k^{3}-i \theta k^{3}\right) \\
& +\left(s^{2}+\omega_{0}^{2}+2 \nu k^{2} s\right)\left(s^{2}+2 \nu k^{2} s+\varepsilon k^{2} l-i \theta k^{3}\right)=0,
\end{aligned}
$$

where the functions $l(k, s), \omega_{0}^{2}(\mathbf{k}), \theta(\mathbf{k})$ and the elastic constant of the surfactant $\varepsilon[4]$ are defined as follows:

$$
\begin{gathered}
\varepsilon \equiv \frac{\Gamma \beta}{\rho} ; \quad l^{2}=k^{2}+\frac{s}{\nu}, \quad \nu=\frac{\mu}{\rho}, \quad \operatorname{Re}(l)>0 \\
\theta(\mathbf{k}) \equiv \frac{1}{\rho}\left(\frac{c_{\mathrm{n}} \Gamma}{1+2 \pi c_{\mathrm{n}} \Gamma k}-\frac{c_{\mathrm{t}} \Gamma}{1-2 \pi c_{\mathrm{t}} \Gamma k}\right) \frac{H_{\alpha} k^{\alpha}}{k} H_{3} \\
\frac{\rho \omega_{0}^{2}}{k}=\rho g \\
+\left(\gamma_{0}-\beta \Gamma+\frac{\Gamma c_{\mathrm{t}}}{2} H_{\mathrm{t}}^{2}-\frac{\Gamma c_{\mathrm{n}}}{2} H_{\mathrm{n}}^{2}\right) k^{2} \\
+\left(\frac{\Gamma c_{\mathrm{n}}}{1+2 \pi k \Gamma c_{\mathrm{n}}}-\Gamma c_{\mathrm{t}}\right)\left(H_{\alpha} k^{\alpha}\right)^{2}+\frac{\Gamma c_{\mathrm{t}} H_{\mathrm{n}}^{2} k^{2}}{1-2 \pi k \Gamma c_{\mathrm{t}}} .
\end{gathered}
$$

3. Stability conditions. The equilibrium solution is stable if and only if $\operatorname{Re}\left(s\left(k_{1}, k_{2}\right)\right)<0$ for any solution $s\left(k_{1}, k_{2}\right)$ of Eq. (20) .

If $H_{\mathrm{n}}, H_{\mathrm{t}}=0, \theta=0$ and Eq. (20) is analogous to the dispersion equation for capillary-gravity waves in the presence of a surfactant but in the absence of magnetic fields [4]. The only difference is the expression for $\omega_{0}^{2}$ that may be negative even for a positive surface tension in the presence of magnetic fields. The stability condition, in this case, is

$$
\forall k_{1}, k_{2}: \omega_{0}^{2}(\mathbf{k})>0
$$

The condition (24) is equivalent to one of the simpler conditions $H_{\mathrm{n}}<H_{*}$ or $H_{\mathrm{t}}<H_{*}$, where $H_{*}$ is the critical value of the magnetic field strength depending on other parameters of the problem. For $H_{\mathrm{n}} \neq 0, H_{\mathrm{t}}=0$ and three different values of $\delta=-\lambda_{\mathrm{t}} / \lambda_{\mathrm{n}}$ the dimensionless

$$
q^{*}=\frac{\lambda_{\mathrm{n}} H_{*}^{2}}{2\left(\gamma_{0}-\beta \Gamma\right)}
$$

is shown in Fig. 1. The function $q^{*}(b)$ is defined by the following parametric expressions:

$$
\delta q^{*}=t+2 t^{2}+t^{3}, \quad b\left(1-q^{*}\right)=t^{2}+2 t^{3}, \quad t>0 .
$$

For $H_{\mathrm{n}}=0, H_{\mathrm{t}} \neq 0$, the critical value of the magnetic field strength is defined by the equation

$$
\gamma_{0}-\beta \Gamma+\Gamma c_{\mathrm{t}} H_{*}^{2} / 2=0 .
$$




\section{A.V. Zhukov}

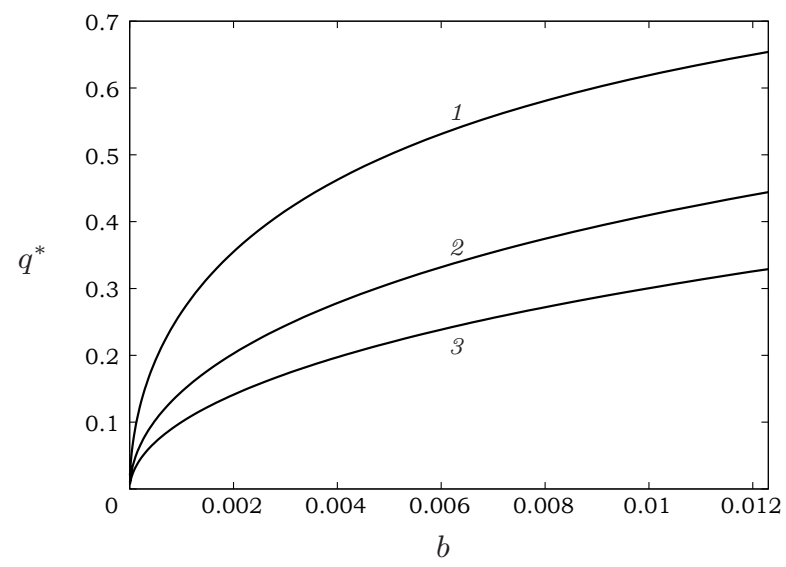

Fig. 1. The critical dimensionless magnetic field strength $q^{*}=\lambda_{\mathrm{n}} H_{*}^{2} / 2\left(\gamma_{0}-\beta \Gamma\right)$ vs. the parameter $b=\left(\gamma_{0}-\beta \Gamma\right) / \rho g\left(2 \pi \lambda_{\mathrm{t}}\right)^{2}$ at $\delta=0.1(1), 0.2(2), 0.3(3)$.

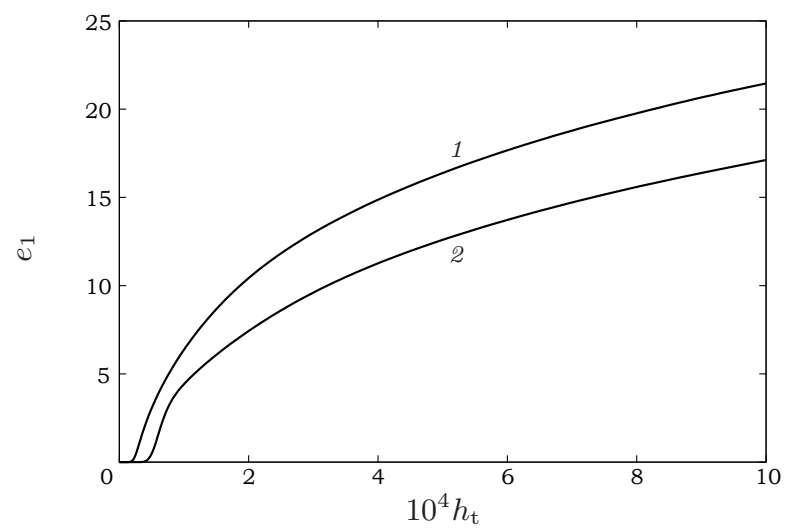

Fig. 2. The dependence of $e_{1}=\varepsilon_{*} \rho / \sigma_{*}$ on the dimensionless tangential component of the external magnetic field $h_{\mathrm{t}}\left(h_{\mathrm{t}}^{2}=\lambda_{\mathrm{n}} H_{\mathrm{t}}^{2} / \sigma_{*}\right)$, where $\sigma_{*}=\gamma_{0}-\beta \Gamma-\lambda_{\mathrm{n}} H_{\mathrm{n}}^{2} / 2+\lambda_{\mathrm{t}} H_{\mathrm{t}}^{2} / 2$ for the parameters $\lambda_{\mathrm{n}} H_{\mathrm{n}}^{2} / \sigma_{*}=1, \nu^{2} \rho / \sigma_{*} \lambda_{\mathrm{n}}=0.1, \rho g \lambda_{\mathrm{n}}^{2} / \sigma_{*}=10^{-6}$ and $\delta=0.1(1), \delta=1$ (2).

Note then that the stability condition requires $c_{\mathrm{t}}<0$ [3].

If $H_{\mathrm{n}}, H_{\mathrm{t}} \neq 0$, the condition (24) is insufficient for the stability of the liquid surface. In that case, the stability condition is equivalent to $\varepsilon>\varepsilon_{*}\left(H_{\mathrm{t}} / H_{\mathrm{n}}\right)$, where the critical value $\varepsilon_{*}$ depends on the other parameters of the problem (see Fig. 2).

4. Conclusions. The stability of the capillary-gravity wave motion on the horizontal free surface of a viscous incompressible fluid in the presence of a magnetic surfactant in an external magnetic field was studied taking into account the anisotropy of the surface tension tensor and the dependence of the surface tension on the surfactant surface density and on the magnetic field strength. It is shown that for a normal as well as for a tangential external magnetic field the horizontal free liquid surface is unstable for the field strength exceeding some critical value that does not depend on the elastic constant of the surfactant film. However, for an oblique external magnetic field, the stability of the free surface depends not only on the field value but also on the surfactant elastic constant. 
Stability of capillary-gravity waves in the presence of magnetic surfactant in an ...

Acknowledgements. Support by the Russian Foundation for Basic Research (projects Nos. 16-01-00157 and 17-01-00037) is acknowledged.

\section{References}

[1] P. Brown, T.A. Hatton, J. Eastoe. Magnetic surfactants. Curr. Opinion in Colloid and Interface Science, vol. 20 (2015), pp. 140-150.

[2] A.N. Golubyatnikov, G.I.Subhankulov. On the surface tension of a magnetic fluid. Magnetohydrodynamics, vol. 22 (1986), no. 1, pp. 62-67.

[3] A.N. Golubyatnikov. Thermodynamic instability of anisotropic magnetic fluids and their surfaces in a magnetic field. Advances in Mechanics, vol. 4 (2006), no. 3, pp. 3-25 (in Russian).

[4] V.G. Levich. Physicochemical Hydrodynamics (Prentice-Hall, 1962), 700 p.

Received 27.12.2017 